\title{
The Purpose and Definition of the Minimum Magnitude Limit in PSHA Calculations
}

\section{by Julian J. Bommer and Helen Crowley}

\begin{abstract}
A lower limit of magnitude $M_{\min }$ is routinely defined for integrations of earthquake scenarios in probabilistic seismichazard analysis but there is widespread misunderstanding of the technical bases for determining the value of this parameter. In this article, several misconceptions are identified and discarded prior to providing a clear and unambiguous definition of $M_{\min }$ that points to the fact that seismic-hazard assessment is always conditioned by the intended application of the outputs. We argue that $M_{\min }$ is therefore an engineering parameter that is ultimately related to seismic risk rather than seismic hazard. The confusion surrounding this topic could be largely alleviated by defining lower limits on appropriate intensity measures rather than on magnitudes used as a proxy for shaking levels.
\end{abstract}

\section{INTRODUCTION}

Although it may seem unusual to begin a paper with an apology, we recognize that for many readers the issues discussed in this article will seem blindingly obvious and unworthy of publication in a reputable journal. However, our experience suggests that there is actually a great deal of confusion regarding the minimum magnitude considered in probabilistic seismic-hazard analyses (PSHAs), a parameter generally referred to as $M_{\min }$. We concluded that it is therefore worthwhile to briefly discuss the definition and purpose of $M_{\min }$, and to address some of the misconceptions that we encountered regarding this parameter. The discussion raises important questions of the intimate relationship-albeit often implicit rather than declared-between seismic-hazard analysis and the quantification of seismic risk. This in turn leads us to discuss alternative approaches for meeting the objectives associated with the specification of $M_{\min }$ in PSHA.

Although the motivation for writing this article has come from innumerable encounters with misunderstandings or misinterpretations of the concept of $M_{\min }$, we carefully avoid identifying any specific cases by name or reference. Our objective is to try to clarify some fundamental issues related to this parameter while also exploring some of the broader issues that it raises regarding the assessment of seismic hazard and risk.

\section{INTEGRATION LIMITS IN PSHA}

In essence, PSHA is the integration over distributions of three random variables that characterize the seismic source model, which defines the location and magnitude of hypothetical earthquakes scenarios, and the predicted ground-motion amplitudes at the site under study due to each of these earthquakes (Fig. 1). The variables are magnitude $(\mathbf{M})$, source-to-site distance $(R)$, and the number of standard deviations $(\sigma)$ above or below the logarithmic mean value from the ground-motion prediction equation (GMPE); this last variable is usually represented by the letter $\varepsilon$. Depending on the nature of the seismic source models and the GMPEs, the PSHA calculations may also include integration over additional distributions of random variables such as focal depth, style of faulting, and the strike, dip, and rake of fault ruptures, whether these are real or virtual (e.g., Monelli et al., 2014).

For each of the random variables in the PSHA calculations, consideration must be given to the limits of integration, which may have a theoretical or practical basis. For features of the earthquake source such as focal depth, the limits will be defined by the crustal characteristics and will correspond in general to the top and bottom of the seismogenic layer (and these limits may also vary with magnitude to preclude, e.g., large-magnitude earthquakes initiating very close to the Earth's surface). For style of faulting and rupture plane geometries, the range of possibilities is obvious and the local geological structure may lead to narrower limits. The issue of the greatest interest is the limits on the three key variables of $\mathrm{M}, R$, and $\varepsilon$, and each of these is now considered in turn.

The distribution of logarithmic residuals associated with a GMPE is generally represented by a Gaussian distribution and therefore it is theoretically unbounded. However, for practical reasons limits are applied. In terms of negative values of $\varepsilon$, truncating at one or two standard deviations below the mean will have almost no impact on hazard calculations because negative values of this variable only contribute to hazard estimates at very high annual frequencies of exceedance (AFE), if they contribute at all. At the upper end, a value of 6 is widely used; the probability of higher exceedances of the logarithmic mean value is vanishingly small and hence greater values would not contribute noticeably to the hazard estimates. Nonetheless, it is not uncommon in practice to encounter PSHA being calculated with the ground-motion residuals truncated at values of 
(a)

(b)

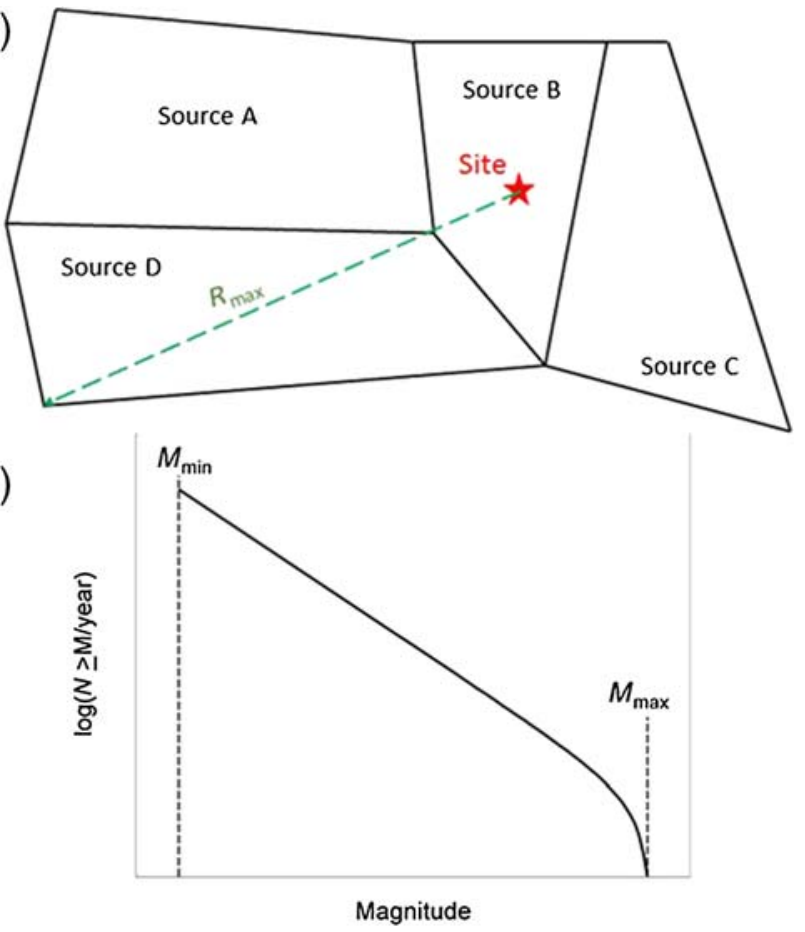

(c)

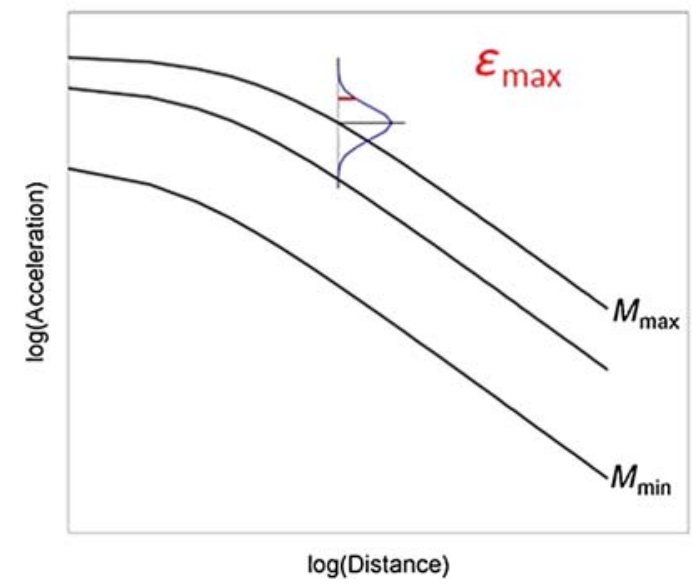

A Figure 1. Schematic illustration of probabilistic seismic-hazard analysis calculation using area source zones of distributed seismicity, indicating the integration limits on the main random variables that are integrated in the estimation of the annual exceedance frequency of a given level of acceleration: (a) maximum distance to limits of seismic sources, (b) minimum and maximum magnitudes, and (c) maximum number of standard deviations of the ground-motion prediction equation. The color version of this figure is available only in the electronic edition.

$\varepsilon=3$ or even lower, which can have a pronounced effect on the estimated hazard, particularly at low AFEs. However, there is neither physical nor statistical justification for such low truncations (Electric Power Research Institute [EPRI], 2006a; Strasser et al., 2008).

In terms of distance, the minimum value considered will depend on the type of seismic source and the distance metric used in the GMPE. For fault sources, the closest approach of the fault to the site will define $R_{\min }$. For diffuse seismicity in area source zones, $R_{\min }$ will generally correspond to an earthquake initiating directly below the site and will take a value depending on the depth distribution and the distance metric; for epicentral $\left(R_{e p i}\right)$ and Joyner-Boore $\left(R_{\mathrm{JB}}\right)$ distances, $R_{\text {min }}$ will be zero.

The maximum value of distance considered depends primarily on the relative levels of seismic activity in the host source zone (for diffuse seismicity) or the closer seismogenic faults, and the activity levels of more distant sources. In cases where there are no significant sources of seismicity with far greater levels of activity than those in close proximity to the site, significant hazard contributions will be limited to at most 200-300 km depending on the attenuation rates. For lower oscillator frequencies $R_{\max }$ will tend to be larger, and for low AFEs the tendency will be for $R_{\max }$ to reduce as the hazard estimates are more dominated by nearby contributions. Exceptions to these general patterns include locations inland from major subduction zones and sites in seismically quiet areas where there is a significant source of seismicity at a greater distance from them. An example of the latter might be a site in southeast United States where for low AFEs and low oscillator frequencies, the New Madrid source dominates. An advisable approach is to initially define sources extending $300 \mathrm{~km}$ or more from the site-and farther if there is a remote but highly active source of seismicity - and then reducing the upper limit of the integrations if disaggregations indicate null contributions beyond a certain limit.

Magnitude scales are, at least in theory, unbounded, being based on the logarithmic value of wave amplitudes recorded on sensitive instruments. In practice, there is an apparent upper limit to all magnitudes except moment magnitude $\mathbf{M}$ because of saturation; there is also a physical upper limit because an earthquake of moment magnitude close to 10 would need to split the Earth's crust in two. $M_{\max }$, as it is defined for PSHA and used here, is a time-dependent upper bound. $M_{\max }$ represents the largest earthquake a particular seismic source is capable of generating over the defined time frame and hence it is primarily limited by the maximum dimensions of potential fault ruptures and generally cannot be defined from statistical analysis of earthquake catalogs, although such approaches have been advocated (e.g., Kijko, 2004). The maximum magnitude considered in PSHA $\left(M_{\max }\right)$ is a parameter that has received considerable attention and has been the subject of several studies, particularly for stable tectonic regions such as the central and eastern United States (Johnston et al., 1994; Wheeler, 2009; U.S. Nuclear Regulatory Commission [USNRC], 2012). Hazard estimates are generally not strongly sensitive to $M_{\max }$ except at the low AFEs relevant to the seismic design and assessment of safety-critical structures, and particularly for spectral accelerations at lower oscillator frequencies. Bender and Campbell (1989) found that the influence of $M_{\min }$ is almost exactly the opposite of that of $M_{\max }$. More recently, there has been focus on $M_{\max }$ for induced seismicity (e.g., Bommer and van Elk, 2017), where its influence may be more significant.

Magnitude scales also have no absolute lower limit and microearthquakes are routinely assigned negative values. 
However, to integrate hazard contributions from earthquakes of magnitude -3 and larger would involve pointlessly laborious computations-particularly if using Monte Carlo approaches to PSHA (e.g., Musson, 2000; Assatourians and Atkinson, 2013) - and therefore there is a practical reason for imposing a lower limit. However, the basis for selecting this lower threshold $M_{\min }$ is a topic about which there appears to be widespread confusion.

\section{THINGS THAT $M_{\text {MIN }}$ IS NOT}

Before providing a clear definition of $M_{\min }$, we briefly list various attributes that have been given to $M_{\text {min }}$ that misrepresent its purpose and meaning. We have not made up any of these definitions-these are all explanations for $M_{\min }$ that we have read or heard from seismologists and earthquake engineers:

1. The value of $M_{\min }$ is not chosen to make the hazard calculations simpler or quicker. Although for practical reasons a lower bound on magnitude is required for the integrations - and the higher this value, the more efficient the calculations-this alone is not an appropriate criterion for fixing the value.

2. $M_{\min }$ is neither the lower limit of completeness of the earthquake catalog nor the smallest magnitude used in the fitting of recurrence relationships. This is one of the most common confusions regarding $M_{\min }$. The smallest magnitude considered in the determination of the Gutenberg-Richter $a$ - and $b$-values may be equal to $M_{\min }$ but it may also be smaller or larger than $M_{\text {min }}$; it does not determine the value of $M_{\min }$.

3. $M_{\min }$ is not the minimum magnitude at which the chosen GMPEs are applicable. $M_{\min }$ may be smaller than the lower limit of applicability of the GMPEs and if that is the case, the hazard analyst faces a challenge because empirical GMPEs often do not extrapolate reliably to smaller magnitudes, and indeed may often be unreliable even at the lower limit inferred from the distribution of the underlying data (e.g., Bommer et al., 2007). Like other challenges in performing a PSHA, it needs to be addressed-applying the GMPEs down to magnitudes as small as $M_{\min }$-which may require the analyst to either take into account the additional epistemic uncertainty or to conduct additional studies to calibrate the equations. On this point, there is also a potential circularity of which it is valuable to be aware: the lower magnitude limit of many GMPEs reflects commonly used $M_{\min }$ values in PSHA practice, but this should not automatically determine the $M_{\min }$ adopted for any particular application. In this context, it is worth noting that in recent years there has been a trend towards including smaller-magnitude recordings in the derivation of GMPEs, which has led to lower predicted amplitudes at smaller magnitudes (e.g., Chiou et al., 2010; Abrahamson et al., 2014).

4. $M_{\min }$ is also not set to ensure that sufficient hazard is estimated. We refer here to statements that a high value of $M_{\text {min }}$ leads to underestimation of the hazard, a statement which misses the point that the purpose of $M_{\min }$ is specifically to exclude certain contributions from the hazard estimate (more on this in the Definitions of $M_{\min }$ section ). A particular case of this argument, which we met many times, is that $M_{\min }$ needs to be set to a low value for PSHA in regions of low seismicity, as if more earthquakes need to be considered in the small-magnitude range to compensate for the scarcity or absence of larger earthquakes. In fact, although extremely unlikely occur in any real situation, it is theoretically conceivable for there to be a case in which $M_{\min }$ assumes a larger value than $M_{\max }$, leading to a null hazard estimate.

In dismissing these misunderstandings regarding $M_{\min }$, two important issues emerge. The first is that although PSHA attempts to characterize the threat posed by seismic ground shaking at a particular location, it should not be regarded as a way to determine the hazard as if this were some absolute and immutable quantity. This brings us to the second issue, which follows directly from this first assertion: the way PSHA calculations are performed is not-and should not be-divorced from how the hazard estimates are to be used.

\section{WHAT $M_{\text {MIN }}$ IS}

Having identified some of the common misunderstandings regarding $M_{\min }$, in this section we offer definitions of this parameter, the clearest of which links $M_{\min }$ to seismic risk.

\section{Definitions of $\boldsymbol{M}_{\min }$}

To some extent, the degree of confusion regarding $M_{\min }$ (sometimes referred to as $m_{0}$ ) is surprising given that several fairly clear statements regarding the meaning of this parameter have been made in the literature. For example, the landmark paper by Cornell (1968, p. 1586) that set out the basic formulation for PSHA:

....and $m_{0}$ is some magnitude small enough, say 4 , that events of lesser magnitude may be ignored by engineers.

In their significant note on the issue of $M_{\text {min }}$, Bender and Campbell (1989, pp. 199 and 202) made the following statements:

\section{...high peak accelerations from small magnitude earth- quakes may not cause significant damage to well-engi- neered structures... and therefore it is arguable whether ground motions from small-magnitude earthquakes should be included in seismic hazard calculations...... If $a_{\max }$ [peak ground acceleration at a particular return period] is used in decisions relating to the seismic safety of a structure, then only potentially 'damaging' earth- quakes should be included in the hazard analysis.}

Numerous statements conveying the same rationale can be found in basic texts on engineering seismology and earthquake engineering, such as in Reiter (1990, p. 201): 
The lower bound, or minimum, magnitude represents that level of earthquake size below which there is no engineering interest.

Kramer (1996, p. 123) makes the following statements:

For engineering purposes, the effects of very small earthquakes are of little interest and it is common to disregard those that are not capable of causing significant damage..........In most PSHAs, the lower threshold magnitude is set at values from about 4.0 to 5.0 since magnitudes smaller than that seldom cause significant damage.

McGuire (2004, p. 39) makes the following assertions regarding $M_{\min }$ :

The lower bound, $m_{\min }$, is chosen on the basis of the minimum magnitude that will cause damage or loss and that must be considered for risk mitigation purposes.

Although these quotes express the idea in different waysand use different symbols-the basic idea that they convey is consistent. The concept could be stated in the following way: $M_{\min }$ is the smallest magnitude of earthquake that is capable of generating potentially damaging levels of ground shaking. Immediately, it is important to stress that $M_{\min }$ is therefore a necessary but not sufficient condition for damaging ground motions; in other words, the fact that an earthquake has a magnitude equal to or greater than $M_{\min }$ does not mean that the motions it generates will be damaging, but it does assume that earthquakes smaller than $M_{\text {min }}$ will never be damaging.

This definition, however, instantly evokes questions: Damaging to what? And what constitutes damage? Clearly the $M_{\min }$ value for minor cracking in unreinforced masonry dwellings and the $M_{\min }$ for inelastic response of a nuclear reactor containment building would be quite different. Consequently, we believe that a more complete and less ambiguous definition of $M_{\min }$ would be as follows: $M_{\min }$ is the lower limit of integration over earthquake magnitudes such that using a smaller value may result in higher estimates of seismic hazard but would not alter the estimated risk to the exposure under consideration. Risk estimation in this framework must be probabilistic, yielding the annual frequency or probability of exceeding a defined limit state (i.e., performance target).

Although perhaps a little cumbersome, this proposed definition fully captures the purpose and meaning of $M_{\min }$. It also highlights the fact that this input to PSHA is essentially an engineering parameter related to risk.

\section{Commonly Used Values of $\boldsymbol{M}_{\text {min }}$}

A review of practice reveals that values of $M_{\text {min }}$ do indeed generally lie in the 4-5 range as indicated in the earlier quote from Kramer (1996), although clearly the interpretation of the exact limits depends on the magnitude scale employed. In terms of general applications for engineering design of standard structures, one can look at PSHA studies conducted for the purpose of seismic-hazard mapping. For the U.S. Geological Survey (USGS) seismic-hazard maps, values of $\mathbf{M} 5.0$ for the western United States (increased to 6.5 for deep subduction earthquakes in Oregon) and $\mathbf{M} 4.7$ in the eastern United Stateswhere traditionally a value of 5 on the $m_{b L g}$ scale has been used-are adopted (Petersen et al., 2014), whereas the Seismic Harmonization in Europe (SHARE) seismic-hazard map for Europe was calculated with a lower magnitude limit of M 4.5 (Woessner et al., 2015). For the U.K. seismic-hazard map, Musson and Sargeant (2007) note that M 5.0 is considered appropriate for dams and nuclear power plants (NPPs) and $\mathbf{M} 4.0$ for some other applications, leading to their selection of $\mathbf{M} 4.5$ as an appropriate compromise.

The threshold of $\mathbf{M} 5$ often adopted in PSHAs conducted for NPP sites is worthy of some comment because it is the most thoroughly considered selection of a threshold magnitude value for PSHA. The value of 5 for $M_{\text {min }}$ was recommended in EPRI (1989), a 360-page document arising from a three-day workshop conducted by the EPRI specifically "to establish a lower-bound earthquake magnitude, below which the potential for damage to nuclear plants is negligible." The approach that EPRI (p. iii) adopted to address this goal was summarized as follows:

\section{The objective of the workshop was to consider a broad range of issues that could provide insight to the engineer- ing significance of ground motion generated by small- magnitude earthquakes. Based on the presentations at the meeting it was intended to develop a strategy to select a lower-bound magnitude for use in seismic hazard assessments. An Advisory Committee reviewed the infor- mation presented at the workshop and provided recom- mendations concerning the level of earthquake magnitude that may be damaging to nuclear power plant structures and equipment and a strategy to establish a sound basis to determine the lower bound magnitude.}

The value of $\mathbf{M} 5$ has since been widely adopted in the nuclear industry and is reflected in the guidance of the International Atomic Energy Agency (IAEA), which states in its current guidance on seismic-hazard assessments for nuclear facilities that "a selected lower bound magnitude should not exceed $M_{\mathrm{w}}=5.0$ " (IAEA, 2010). This is a good point to reflect on in relation to misconception 4 listed earlier, namely that $M_{\min }$ should be set to lower values in seismically quiet regions. Although it is clearly possible for the design accelerations for an NPP to be lower in a region of lower seismicity, it is clearly unthinkable that an NPP should be constructed that could be threatened by an earthquake of magnitude 4.5, even occurring in very close proximity.

Although the threshold of $\mathbf{M} 5$ for $M_{\min }$ in PSHA for nuclear sites is widely accepted, this value may not be appropriate for other applications, as has already been indicated. In the generation of one-year seismic-hazard forecasts due to induced and natural earthquakes, the USGS originally considered an $M_{\min }$ of $\mathbf{M} 4.7$ but later reduced this to $\mathbf{M} 4.0$ for subsequent sensitivity analyses "because lower magnitude earthquakes have caused damaging ground shaking” (Petersen et al., 
2017, p. 773). Another example related to relatively small induced earthquakes, whether due to hydraulic fracturing or enhanced geothermal systems, would be cases where the risk is quantified as nuisance or disturbance to the exposed population and hence the fragility curves would define the probability of the shaking being felt (Douglas and Aochi, 2014). In such an application, $M_{\min }$ could be very small. This is, however, still entirely consistent with the idea of $M_{\min }$ being a risk-related parameter: if the nuisance caused by the induced shaking were to lead to suspension of the activity causing the seismicity, then there is a consequence (primarily commercial in nature) of the hazard, just as there is in the case of physical damage.

In closing this section, we note that Bender and Campbell (1989) proposed that rather than defining an abrupt magnitude truncation, a tapered transition could be used to define the lower magnitude limit.

\section{The Inextricable Nature of Seismic Hazard and Seismic Risk}

We would contend that outside of the framework of the assessment or mitigation of seismic risk - which could be defined as the probability of adverse effects to population and/or the built environment as a result of earthquake shaking_-seismic-hazard assessment has little or no meaning. When this view has been expressed, two situations have been put forward as counter arguments, the first being that hazard assessments can be used to compare the hazard in two regions or locations. The usefulness of such comparisons is not clear to us unless, for example, it is related to site selection for a critical facility, in which case it does fall within the realm of risk-informed decision making. The second case is a hazard assessment for the design of a strong-motion recording network, to which we would respond that this is also a risk-based assessment, the risk in this case being a poor return on the investment in terms of fewer recordings than might be hoped for otherwise. All PSHA studies conducted for anything other than academic curiosity or global mapping purposes as, for example, in the Global Seismic Hazard Assessment Program (GSHAP) project (Giardini, 1999) are related to risk assessment whether the study is conducted to determine insurance and reinsurance policies for a portfolio of high-value buildings or industrial facilities, to produce zonation maps for seismic design codes, or for the site-specific design of an NPP.

To illustrate our point, we may consider a region with an old and deteriorated building stock constructed without consideration of lateral resistance to earthquake loads. For such a case, a seismic source model will be developed, an appropriate GMPE selected (or, more likely, logic trees would be constructed for both of these inputs), and a hazard model developed as the basis for a risk assessment. In this case, the selected value of $M_{\min }$ may be rather low. In the case of induced earthquakes occurring in a region of previously very low seismicity, it may be even smaller (e.g., Bourne et al., 2015). However, if seismic design criteria were to be established for new reinforced concrete moment-resisting frame buildings in the same region, it would be appropriate to perform the PSHA- possibly using the same seismicity and ground-motion models, but with a higher value of $M_{\min }$.

\section{ALTERNATIVES TO $\boldsymbol{M}_{\text {MIN }}$}

If one accepts the premise that $M_{\text {min }}$ is a risk-related parameter, a question that arises is whether it is the most appropriate and efficient way to achieve the intended goal of avoiding inflation of the hazard estimates by scenarios that do not contribute to the risk. To answer this question, it is helpful to first consider exactly what $M_{\min }$ is intended to do, which is to exclude from the hazard integrations any high-amplitude, short-duration motions that are not sufficiently energetic to be potentially damaging. There are numerous examples of nondamaging motions with high peak ground accelerations (PGA), and for illustration one may consider the accelerogram with a horizontal peak of $0.8 \mathrm{~g}$ recorded during a magnitude $M_{\mathrm{L}} 4.4$ earthquake associated with an enhanced geothermal system in El Salvador (Bommer et al., 2006). Despite this large amplitude, the shaking-which lasted for barely one second-caused no distress or damage to the highly vulnerable rural dwellings in the immediate vicinity.

The focus on the nature of the motions rather than the size of the earthquake is reflected in the following quote from Reiter (1990, p. 203):

\section{If, for example, probabilistic seismic hazard analysis were sufficiently sophisticated to allow separation be- tween non-damaging peak accelerations from small earthquakes and more damaging peak accelerations from large earthquakes, lower bound magnitudes could be extended as low as the data allowed.}

Such an approach, based on a ground-motion filter rather than a magnitude cutoff, is precisely what is involved in the use of cumulative absolute velocity (CAV) filters as an alternative to $M_{\min }$. Based on values of CAV predicted as conditional on PGA, all $M-R-\varepsilon$ contributions leading to motions that fall below a specified level of CAV are excluded from the calculation of AFE of PGA and spectral accelerations (EPRI, 2006b). Excluding nondamaging motions is clearly a more rational approach than an abrupt, distance-independent cutoff on magnitude. In the 50.54(f) letter issued on the first anniversary of the Fukushima accident in which the Nuclear Regulatory Commission requested that all U.S. NPP owners update their seismic-hazard assessments, the following instructions:

\section{To remove non-damaging lower-magnitude earth- quakes, addressees should either use a lower bound magnitude cutoff of moment magnitude $\left(M_{\mathrm{w}}\right) 5$ or the cumulative absolute velocity (CAV) filter for the PSHA. The CAV filter should be limited to $M_{\mathrm{w}}$ less than or equal to 5.5. (USNRC, 2012, p. 9)}

In essence, the use of a CAV filter prevents ground motions of low energy from contributing to the hazard estimate. The original intent of the CAV parameter was related to seismic monitoring at NPPs and decision making related to safe 


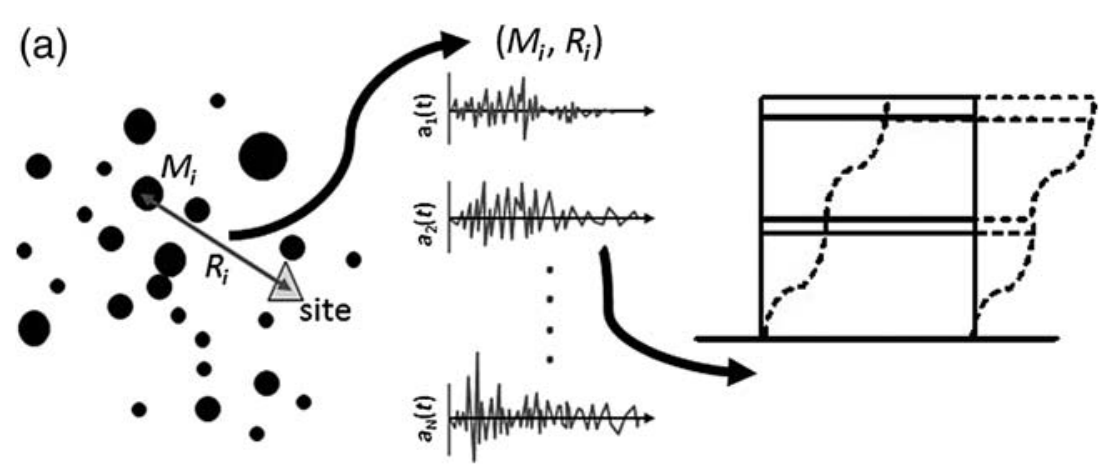

(b)

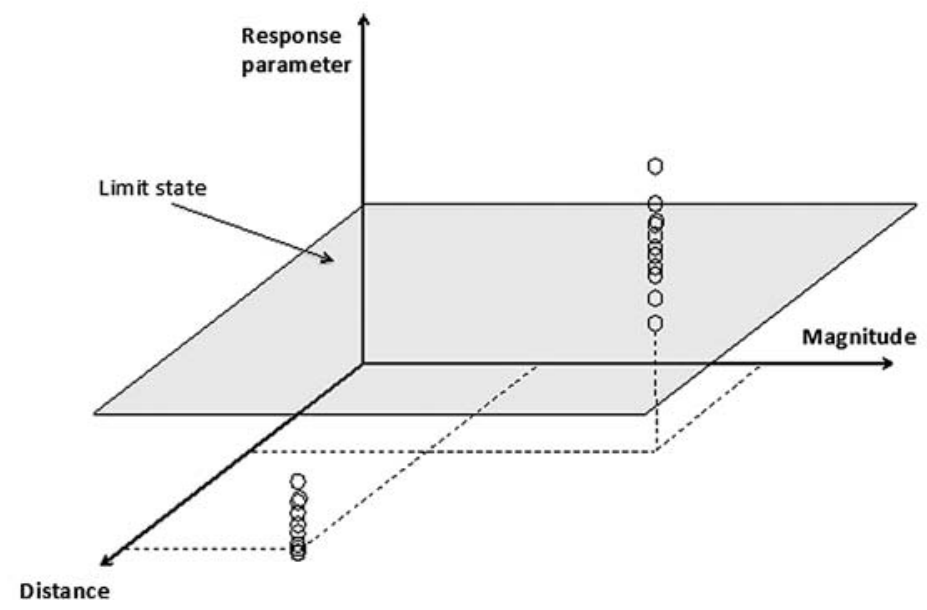

- Figure 2. (a) Schematic illustration of comprehensive seismic risk assessment for a structure located at the site marked in triangle. The dots represent epicenters of a synthetic earthquake catalog (the size being proportional to the magnitude). The response of a model of the structure is calculated from dynamic analyses using a large suite of representative acceleration time histories for each magnitude-distance combination. (b) The resulting structural response metric from each analysis is compared with the level that defines the limit state.

shutdown and restart following earthquake shaking (EPRI, 1988). Nonetheless, the use of CAV filters was proposed as an alternative to $M_{\min }$ (EPRI, 2006b; Watson-Lamprey and Abrahamson, 2007) and these have been widely applied, prompting the development of new GMPEs for the conditional prediction of CAV (Campbell and Bozorgnia, 2010). However, other ground-motion parameters or vectors of parameters might serve the same purpose equally well. Most likely, different parameters may perform better in different applications, depending on which measures of ground-motion intensity are found to be most efficient for defining the fragility functions of the exposure elements for which risk is directly or indirectly being assessed or mitigated.

In light of these points, it becomes clear that $M_{\min }$ was only ever required because of the use of insufficient groundmotion parameters (or intensity measures [IMs]) such as PGA, which can assume large values without the motion having the potential to cause structural damage. The sufficiency of IMs in the context of seismic risk assessment is discussed in the Simplified Risk Assessment section.

\section{$M_{\text {MIN IN SEISMIC RISK }}$ CALCULATIONS}

To fully understand the significance of $M_{\min }$, it is necessary to explore its influence on estimates of seismic risk. We start by explaining how a comprehensive risk assessment would be undertaken from nonlinear dynamic analyses using time histories that cover the full space of magnitude-distance events contributing to the hazard, and then we look at how this is typically simplified to the convolution of hazard curves and precomputed fragility functions.

\section{Comprehensive Risk Assessment}

Risk assessments are usually carried out for a range of metrics, including damage levels, collapse, fatalities, and economic loss. In an analytical risk assessment, each of these metrics needs to be related to the nonlinear response of a numerical model of the structure under assessment, obtained from nonlinear dynamic analyses.

In an ideal world, the following procedure could be followed to perform the risk assessment for an individual structure. Monte Carlo simulations could be used to generate a catalog of earthquake scenarios (defined by magnitude and location, and possibly also style of faulting and other source-related parameters) of sufficient length to sample the lowest probabilities of interest in the risk calculations. For each earthquake, a large suite of acceleration time histories could then be selected or simulated to be consistent with the magnitude $M$ and the distance $R$ from the site. Dynamic analyses could then be run on a suitable structural model using each accelerogram (Fig. 2); collectively, the time histories would sample the range of possible ground motions for such a scenario in terms of amplitude, frequency content, and duration or number of cycles. The response of the structure under each accelerogram is measured in terms of a parameter such as drift, and the results could then be plotted as shown schematically in Figure 2. Assuming the limit state of interest-for example, the nearcollapse condition - can be unambiguously defined by a single value of the chosen response parameter, then the frequency or probability of reaching or exceeding the limit state can be estimated from the number of data points above the threshold as a proportion of the total number of analyses. This probability of exceedance, together with the annual frequency of occurrence of all earthquake events, can be used to estimate risk metrics such as the average annual probability of limit state exceedance. The average annual probability of structural collapse is currently being used as a metric to calibrate seismic design codes in the United States (Luco et al., 2007). As shown in Figure 2, there will be some magnitude-distance combina- 
tions for which none of the analyses will lead to structural response exceeding the limit state, and these scenarios therefore do not contribute to the risk at all. Such scenarios may include small-magnitude earthquakes even when they occur very close to the site.

A less computationally intensive approach to the method presented above would be to simulate, for each Monte Carlo scenario, correlated estimates of ground shaking parameters (such as spectral acceleration, CAV, 5\%-75\% significant duration) at the site of the risk assessment using GMPEs and correlation models (see e.g., Bradley, 2010) and then time histories would need to be selected (or most likely simulated) to match these hazard characteristics. Nonlinear dynamic analyses would then be undertaken using these time histories, and the nonlinear response of each record would be obtained. The number of nonlinear dynamic analyses required to obtain stable estimates of structural response would be much smaller than when using records selected or generated only to match the magnitudedistance-site scenario (Hancock et al., 2008). The probability of exceedance of the limit state threshold for each event would then be estimated and the risk metric calculated as before. As in the previous case, it would be possible to identify the magnitude-distance combinations that never lead to limit state exceedance, or that have a very low contribution to the estimated risk, and thus whose removal does not influence the value of the risk metric (with a given threshold).

In both of the above risk assessment methodologies, the removal of earthquake events does not influence the outcome of the risk assessment, but if they were to be removed a priori then this would obviously reduce the computational intensity of the risk assessment. Also, as discussed previously in this article, their removal would influence the hazard curves, and so if hazard products are to be presented alone (e.g., for use in a simplified risk assessment, as discussed in the Simplified Risk Assessment section, or a seismic design code), then these events should also be removed from the hazard calculations. However, it is important to note that different events might be removed for different risk metrics, and so a different hazard curve would result for each application.

\section{Simplified Risk Assessment}

Clearly, the comprehensive approach presented above is never implemented in practice because the computational burden would be excessive and it would be very difficult to obtain such large suites of appropriate accelerograms for every single earthquake scenario. Instead, the number of nonlinear dynamic structural analyses is greatly reduced, using a relatively small number of input motions to derive fragility functions that show the probability of exceeding the limit state under a given level of shaking. The sacrifice in terms of accuracy that is made in this step is to then represent the ground motion by a single parameter, such as spectral acceleration at the fundamental period of the structure or peak ground velocity, on the horizontal axis of the fragility curve. Fragility functions in terms of scalar IMs are therefore produced separately from the hazard
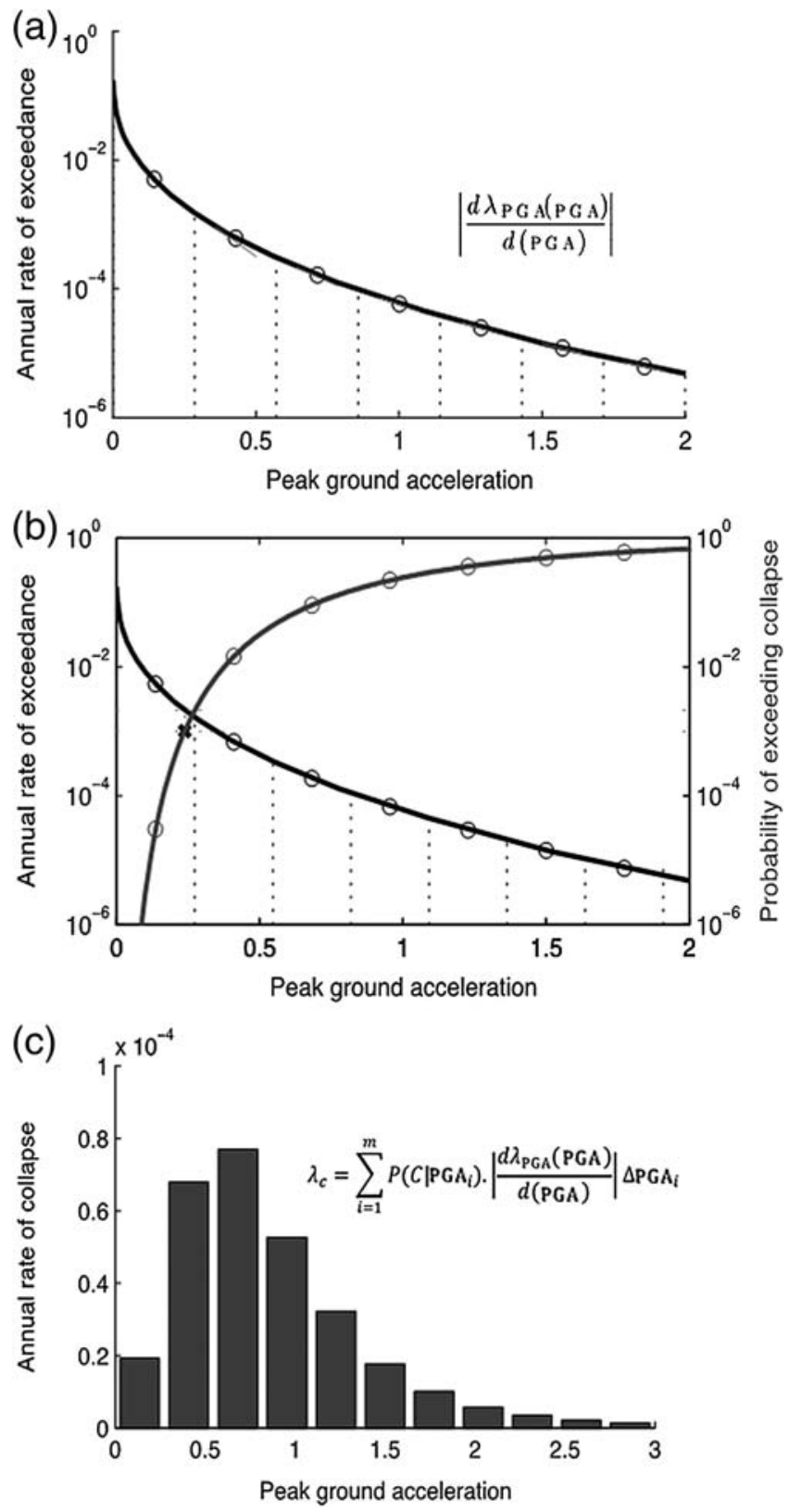

- Figure 3. Illustration of the steps of a standard probabilistic risk analysis: (a) a seismic-hazard curve and the calculation of rate of occurrence of discrete levels of peak ground acceleration (PGA), (b) a fragility function, used to obtain the probability of collapse (or another limit state) at each discrete level of $P G A,(c)$ the convolution of hazard and fragility at each level of PGA, and summed across all PGA levels to give the annual rate of collapse (or another limit state); $\lambda$ represents annual rate.

calculations, and are subsequently convolved with hazard curves in the risk computation (Fig. 3).

To comprehensively represent a fragility function in terms of a scalar ground-motion parameter, it is necessary that other characteristics of the accelerogram (e.g., magnitude, strongmotion duration) do not influence the response of the structural model, and the IM is thus sufficient (see e.g., Luco and 

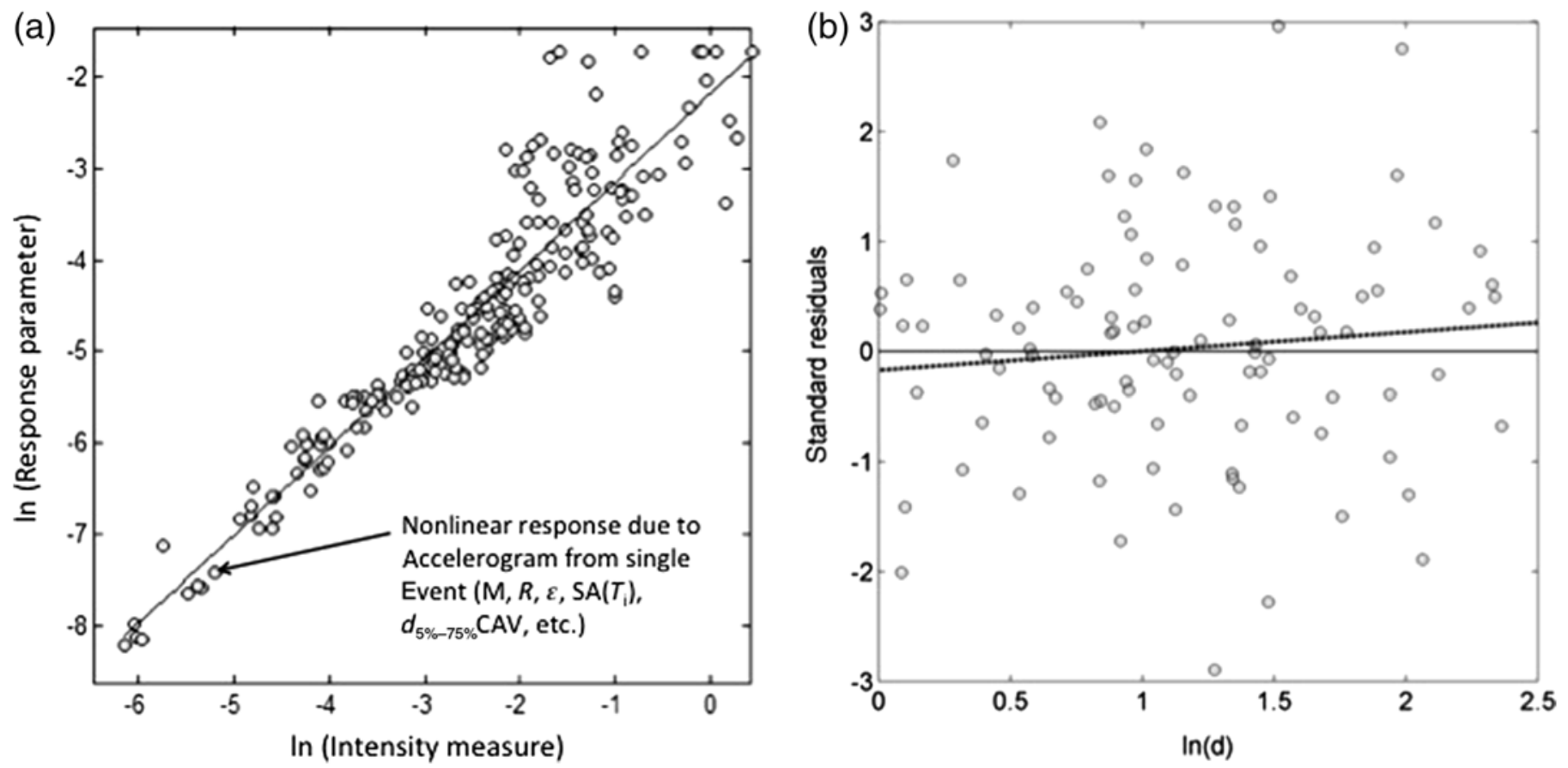

- Figure 4. (a) Illustration of the output of nonlinear analyses from a large set of accelerograms and linear regression in terms of a primary intensity measure (IM); (b) Check of the bias of the residuals of each analysis with respect to a secondary IM (such as duration, $d$ ).

Cornell, 2007). This is usually checked by first applying a linear regression to the data of structural response from each dynamic analysis versus a primary IM (see Fig. 4a), then calculating the residuals of each data point and plotting them against a secondary parameter of the accelerograms (see Fig. $4 \mathrm{~b}$ ). Where a bias with respect to this second parameter is observed, the primary IM is found to be insufficient. This then implies that to adequately characterize the fragility of the structure-for this particular limit state-a vector of ground-motion parameters is required to represent the shaking intensity (e.g., Gehl et al., 2013). The prediction of the second ground-motion parameter for each $M-R$ scenario is conditioned on the prediction of the primary parameter through the correlation of their residuals. For example, a higher-than-average PGA is likely to be associated with a lower-than-average duration (e.g., Bradley, 2011).

In fact, by removing low-magnitude events from the hazard assessment, seismologists have been implicitly assuming (1) that the hazard metric (IM) is a sufficient parameter above the minimum magnitude, and (2) that the events below this threshold do not contribute to the limit state exceedance. For a site-specific PSHA for an important structure, this is something that should actually be confirmed by the engineer. Ideally this might be done through structural analyses using a large number of records to identify the minimum magnitude or preferably a vector of ground-motion parameters, below which the probability of limit state exceedance is extremely low. It is more likely, in practice, to be determined by informed expert judgment. Furthermore, the engineer should then specify whether there are any scalar IMs above this threshold that are sufficient; it is noted that the removal of the low-energy ground motions might indeed make the identification of a sufficient IM for the fragility functions more feasible. Testing for sufficiency generally focuses on recordings from strong (i.e., moderate-to-large magnitude) earthquakes and the conclusions may not hold when extrapolated to smaller events. For example, PGA may be found to be sufficient from magnitude 4.5 to 7 , but a dependence on duration might be found for smaller magnitudes (because motions of very short duration are unlikely to be damaging even if of high amplitude, except perhaps for very weak and brittle structures). If a single sufficient ground-motion parameter cannot be identified, then vectorbased IMs would be needed to ensure sufficiency, despite the additional complexity this may bring to the PSHA, and the vector nature of the hazard should be clearly identified in any stand-alone presentation of the hazard.

\section{DISCUSSION AND CONCLUSIONS}

In this article, we discussed the definition, purpose, and implications of the minimum magnitude considered in PSHA calculations, a parameter usually denoted by $M_{\min }$. The upper magnitude limit considered in PSHA, $M_{\max }$, has been the focus of extensive research and many publications, whereas $M_{\min }$ has received considerably less attention in recent decades. One could question whether the $M_{\min }$ parameter is of sufficient importance to warrant such a lengthy discussion, especially given that its impact on PSHA results is generally limited to relatively high AFEs and to spectral accelerations at higher frequencies, above about $5 \mathrm{~Hz}$ (Beauval and Scotti, 2004), although Grünthal and Wahlström (2001, p. 309) report that lowering $M_{\text {min }}$ leads to 
"drastic increase of the hazard at small mean return periods." We believe, nonetheless, that as one of the indispensable inputs to PSHA and as a parameter around which there is so much confusion, it is worthwhile to attempt some clarification. In the process, we identify that $M_{\min }$ is really an engineering rather than a seismological parameter and its meaning is related to the estimation of seismic risk. Indeed, we conclude that the assessment of seismic hazard cannot be decoupled from risk considerations. Moreover, it does not really make sense to speak of the seismic hazard at a site as if it were a known and absolute quantity; apart from the inevitable epistemic uncertainty, all we have at any moment is an estimate of the hazard based on current knowledge, and calculations conditioned to the intended or potential applications of the results.

With this in mind, we repeat (and simplify because the qualifying reference to hazard is not actually needed) our proposed definition for the minimum magnitude considered in PSHA: $M_{\min }$ is the lower limit of integration over earthquake magnitudes such that using a smaller value would not alter the estimated risk to the exposure under consideration. Adhering to this definition, the largest possible value of $M_{\min }$ should be chosen, which has the attendant benefit of reducing the computational burden of the hazard integral.

If one accepts this definition, then it immediately demands the question of why other integration limits $-\varepsilon_{\min }$ and $R_{\max }$ should not also be defined on the basis of their contributions to risk rather than on levels that do not contribute to the hazard estimate. However, this question is obviated if $M-R-\varepsilon$ contributions are excluded on the basis of thresholds of ground motion-ideally in the form of vectors of parameters-related to the potential contribution to risk. Such an approach would automatically exclude low values of magnitude and epsilon and large distances, because such scenarios would have a negligible contribution to risk estimates.

Although we believe that this is a helpful clarification, we also acknowledge that for applications encompassing a broad exposure such as seismic-hazard mapping for design codes covering a wide variety of structural types, the definition of a single risk-based value of $M_{\min }$ will not be straightforward. This is also the case when multiple performance targets are considered in a code: an appropriate lower threshold for collapse prevention may not be appropriate for damage limitation.

Whether a simple magnitude limit, a combination of minimum magnitude and maximum distance, or a threshold for vectors of ground-motion parameters is used to limit PSHA integrations, all seismic-hazard studies should explicitly state the bounds of the integrations. The PSHA documentation should also identify the intended or potential applications of the results so that risk is correctly quantified, whether implicitly or explicitly.

\section{DATA AND RESOURCES}

No data were used in this study. The source of all quotations is clearly identified in the article and full references are provided. $\mathbf{<}$

\section{ACKNOWLEDGMENTS}

We are grateful to numerous people for discussions on this interesting topic, including all those whose comments alerted us to how commonly misunderstood is the concept of $M_{\min }$, and those with whom we had the opportunity for stimulating exchanges that helped us hone the ideas expressed herein. However, any weaknesses in the arguments put forward in this article are ours and ours alone. We are thankful also to the SRL Associate Editor, Brendon Bradley, Peter Stafford, and an anonymous reviewer for constructive feedback on an earlier version of the article that helped us improve and strengthen the discussions. We also thank Russell Green for clarifying information regarding the effects of small-to-moderate magnitude aftershocks in Christchurch, New Zealand.

\section{REFERENCES}

Abrahamson, N. A., W. J. Silva, and R. Kamai (2014). Summary of the ASK14 ground motion relations for active crustal regions, Earthq. Spectra 30, no. 3, 1025-1055.

Assatourians, K., and G. M. Atkinson (2013). EqHaz: An open-source probabilistic seismic-hazard code based on the Monte Carlo simulation approach, Seismol. Res. Lett. 84, no. 3, 516-524.

Beauval, C., and O. Scotti (2004). Quantifying sensitivities of PSHA for France to earthquake catalog uncertainties, truncation of groundmotion variability, and magnitude limits, Bull. Seismol. Soc. Am. 94, no. 5, 1579-1594.

Bender, B., and K. W. Campbell (1989). A note on the selection of minimum magnitude for use in seismic hazard analysis, Bull. Seismol. Soc. Am. 79, no. 1, 199-204.

Bommer, J. J., and J. van Elk (2017). Comment on "The maximum possible and the maximum expected earthquake magnitude for production-induced earthquakes at the gas field in Groningen, The Netherlands" by Gert Zöller and Matthias Holschneider, Bull. Seismol. Soc. Am. 107, no. 3, doi: 10.1785/0120170040.

Bommer, J. J., S. Oates, J. M. Cepeda, C. Lindholm, J. F. Bird, R. Torres, G. Marroquín, and J. Rivas (2006). Control of hazard due to seismicity induced by a hot fractured rock geothermal project, Eng. Geol. 83, no. 4, 287-306.

Bommer, J. J., P. J. Stafford, J. E. Alarcón, and S. Akkar (2007). The influence of magnitude range on empirical ground-motion prediction, Bull. Seismol. Soc. Am. 97, no. 6, 2152-2170.

Bourne, S. J., S. J. Oates, J. J. Bommer, B. Dost, J. van Elk, and D. Doornhof (2015). A Monte Carlo method for probabilistic seismic hazard assessment of induced seismicity due to conventional gas production, Bull. Seismol. Soc. Am. 105, no. 3, 1721-1738.

Bradley, B. A. (2010). A generalized conditional intensity measure approach and holistic ground-motion selection, Earthq. Eng. Struct. Dynam. 39, 1321-1342.

Bradley, B. A. (2011). Correlation of significant duration with amplitude and cumulative intensity measures and its use in ground motion selection, J. Earthq. Eng. 15, no. 6, 809-832.

Campbell, K. W., and Y. Bozorgnia (2010). A ground motion prediction equation for the horizontal component of cumulative absolute velocity (CAV) based on the PEER-NGA strong motion database, Earthq. Spectra 26, no. 3, 635-650.

Chiou, B., R. Youngs, N. Abrahamson, and K. Addo (2010). Groundmotion attenuation model for small-to-moderate shallow crustal earthquakes in California and its implications on regionalization of ground-motion prediction models, Earthq. Spectra 26, no. 4, 907-926.

Cornell, C. A. (1968). Engineering seismic risk analysis, Bull. Seismol. Soc. Am. 58, no. 5, 1583-1606. 
Douglas, J., and H. Aochi (2014). Using estimated risk to develop stimulation strategies for enhanced geothermal systems, Pure Appl. Geophys. 171, 1847-1858.

Electric Power Research Institute (EPRI) (1988). A criterion for determining exceedance of the operating basis earthquake, EPRI Report No. EPRI NP-5930, Electric Power Research Institute, Palo Alto, California.

EPRI (1989). Engineering characterization of small-magnitude earthquakes, EPRI Report NP-6389, Electric Power Research Institute, Palo Alto, California.

EPRI (2006a). Truncation of the lognormal distribution and value of the standard deviation for ground motion models in the central and eastern United States, EPRI Report 1013105, Electric Power Research Institute, Palo Alto, California.

EPRI (2006b). Use of minimum CAV in determining effects of small magnitude earthquakes on seismic hazard analyses, EPRI Report 1012965, Electric Power Research Institute and US Department of Energy.

Gehl, P., D. M. Seyedi, and J. Douglas (2013). Vector-valued fragility functions for seismic risk evaluation, Bull. Earthq. Eng. 11, 365-384.

Giardini, D. (1999). The Global Seismic Hazard Assessment Program (GSHAP)-1992/1999, Ann. Geofisc. 42, 957-974.

Grünthal, G., and R. Wahlström (2001). Sensitivity of parameters for probabilistic seismic hazard analysis using a logic tree approach, J. Earthq. Eng. 5, no. 3, 309-328.

Hancock, J., J. J. Bommer, and P. J. Stafford (2008). Numbers of scaled and matched accelerograms required for inelastic dynamic analyses, Earthq. Eng. Struct. Eng. 37, no. 14, 1585-1607.

International Atomic Energy Agency (IAEA) (2010). Seismic hazard in site evaluation for nuclear installations, Specific Safety Guide No. SSG-9, International Atomic Energy Agency, Vienna, Austria, 80 pp.

Johnston, A. C., K. J. Coppersmith, L. R. Kanter, and C. A. Cornell (1994). The earthquakes of stable continental regions, 5 Vols., Final Report for Electric Power Research Institute (EPRI), Palo Alto, California, EPRI TR-102261.

Kijko, A. (2004). Estimation of the maximum earthquake magnitude, $M_{\max }$, Pure Appl. Geophys. 161, 1-27.

Kramer, S. L. (1996). Geotechnical Earthquake Engineering, PrenticeHall, Upper Saddle River, New Jersey, USA, 653 pp.

Luco, N., and C. A. Cornell (2007). Structure-specific scalar intensity measures for near-source and ordinary earthquake ground motions, Earthq. Spectra 23, no. 2, 357-392.

Luco, N., B. R. Ellingwood, R. O. Hamburger, J. D. Hooper, J. K. Kimball, and C. A. Kircher (2007). Risk-targeted versus current seismic design maps for the conterminous United States, Proc. of SEAOC2007 Convention, Squaw Creek, California, USA, 26-29 September 2007.

McGuire, R. K. (2004). Seismic hazard and risk analysis, EERI Monograph MNO-10, Earthquake Engineering Research Institute, Oakland, California.

Monelli, D., M. Pagani, G Weatherill, L. Danciu, and J. Garcia (2014). Modeling distributed seismicity for probabilistic seismic-hazard analysis: Implementation and insights with the OpenQuake engine, Bull. Seismol. Soc. Am. 104, no. 4, 1636-1649.

Musson, R. M. W. (2000). The use of Monte Carlo simulations for seismic hazard assessment in the U.K., Ann. Geofisc. 43, no. 1, 1-9.
Musson, R. M. W., and S. L. Sargeant (2007). Eurocode 8 seismic hazard zoning maps for the UK, Technical Report CR/07/125, issue no. 3, British Geological Survey.

Petersen, M., M. Moschetti, P. Powers, C. S. Mueller, K. M. Haller, A. D. Frankel, Y. Zeng, S. Rezaeian, S. C. Harmsen, O. S. Boyd, et al. (2014). Documentation for the 2014 update of the United States national seismic hazard maps, U.S. Geol. Surv. Open-File Rept. 20141091, U.S. Geological Survey, Reston, Virginia.

Petersen, M. D., C. S. Mueller, M. P. Moschetti, S. M. Hoover, A. M. Shumway, D. E. McNamara, R. A. Williams, A. L. Llenos, W. L. Ellsworth, A. J. Michael, et al. (2017). 2017 one-year seismic-hazard forecast for the Central and Eastern United States from induced and natural earthquakes, Seismol. Res. Lett. 88, no. 3, 772-783, doi: $10.1785 / 0220170005$.

Reiter, L. (1990). Earthquake Hazard Analysis: Issues and Insights, Columbia University Press, New York, New York, 254 pp.

Strasser, F. O., J. J. Bommer, and N. A. Abrahamson (2008). Truncation of the distribution of ground-motion residuals, J. Seismol. 12, no. 1, 79-105.

U.S. Nuclear Regulatory Commission (USNRC) (2012). Central and eastern United States seismic source characterization for nuclear facilities, NUREG-2115 Revision 1, U.S. Nuclear Regulatory Commission, Office of Nuclear Regulatory Research, Washington, D.C.

Watson-Lamprey, J. A., and N. A. Abrahamson (2007). Use of minimum CAV in seismic hazard analyses, Proc. of 9 th Canadian Conference on Earthquake Engineering, Ottawa, Canada, 352-358.

Wheeler, R. L. (2009). Methods of $M_{\max }$ estimation east of the Rocky Mountains, U.S. Geol. Surv. Open-File Rept. 2009-1018, U.S. Geological Survey, Reston, Virginia, 441

Woessner, J., D. Laurentiu, D. Giardini, H. Crowley, F. Cotton, G. Grünthal, G. Valensise, R. Arvidsson, R. Basili, M. B. Demircioglu, et al. (2015). The 2013 European seismic hazard model: Key components and results, Bull. Earthq. Eng. 13, no. 12, 3553-3596.

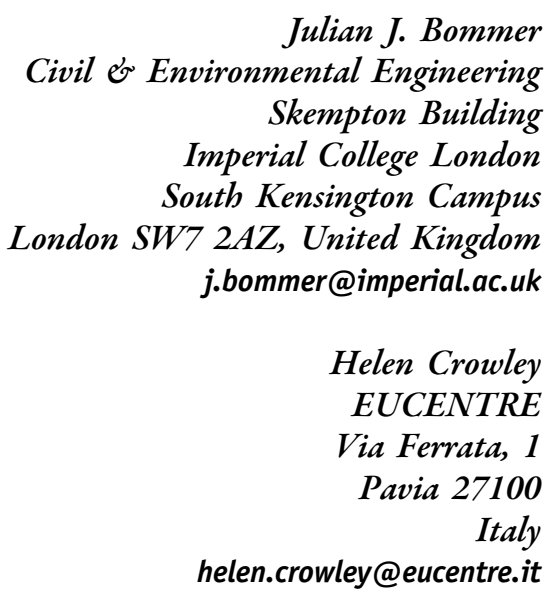

Published Online 10 May 2017 